\title{
Effect of Native Oxide on Reflectivity of Slow and Super Slow Electrons from Mild Steel Surface
}

Š́rka Mikmeková ${ }^{1}$ and Tomohiro Aoyama ${ }^{2}$

${ }^{1}$ Institute of Scientific Instruments, Czech Academy of Sciences, Brno, Jihomoravsky kraj, Czech Republic, ${ }^{2}$ JFE Steel Corporation, Hiroshima, Hiroshima, Japan

Modern commercial scanning electron microscopes (SEMs) are equipped with a cathode lens mode, which enables us to use extremely low landing energies of the primary electrons. There are many benefits of the low energy electron microscopy $[1,2]$ and this technique has been recognized as an important tool for the characterization of advanced steels [3,4]. At lower energies, the contrast in SEM micrographs is more and more influenced by the surface and its state. In this study, we investigated the effect of a native oxide layer on the contrast in SEM images of a mild steel surface obtained at energies from $5 \mathrm{keV}$ up to units of eV. Fig. 1 shows a series of the SEM micrographs of mild steel (Fig. 1 (a)-(i)) collected by an in-house designed ultra-high vacuum scanning low energy electron microscope (UHV SLEEM, Fig. 1 (j)). The micrographs of the same area of view were obtained with various landing energies of the primary beam. Part of the specimen surface was in-situ cleaned by argon ion sputtering to remove the native oxide layer. Fig. 1 (k) shows the Auger spectra collected from the in-situ cleaned and the as-inserted (i.e. native oxide covered) area. After the UHV SLEEM measurement, the specimen was transferred into a commercial SEM and the orientation map was obtained by an electron back-scattered diffraction (EBSD) technique (Fig. 1 (1)). Fig. 2 (a) shows the energy dependence of the contrast between the oxide layer covered and oxide-free area. As visible, the contrast increases approximately linearly with decreasing landing energy up to $30 \mathrm{eV}$. Bellow $30 \mathrm{eV}$, the contrast between the clean and oxidized areas dramatically decreases. This phenomenon is connected with higher penetrability of the native oxide for extremely slow electrons. The energy dependence of the reflected signal from the clean and as-inserted area is shown in Fig. 2 (b). The work function differences $(\Delta \Phi)$ cause a shift of the mirror transition mode. The present work addresses the problem of the use of extremely low landing energies in the SEM for characterization of practical materials and elucidates the effect of the native oxide layer on the reflectivity of slow and super slow electrons from the mild steel surface. The native oxide layer becomes penetrable for super slow electrons and at near-zero landing energies, which is demonstrated through the grain contrast visibility inside the SEM micrographs of the as-inserted area obtained at $0.6 \mathrm{eV}$ landing energy. Presence of the native oxide on mild steel surface results in the work function reduction [5]. 


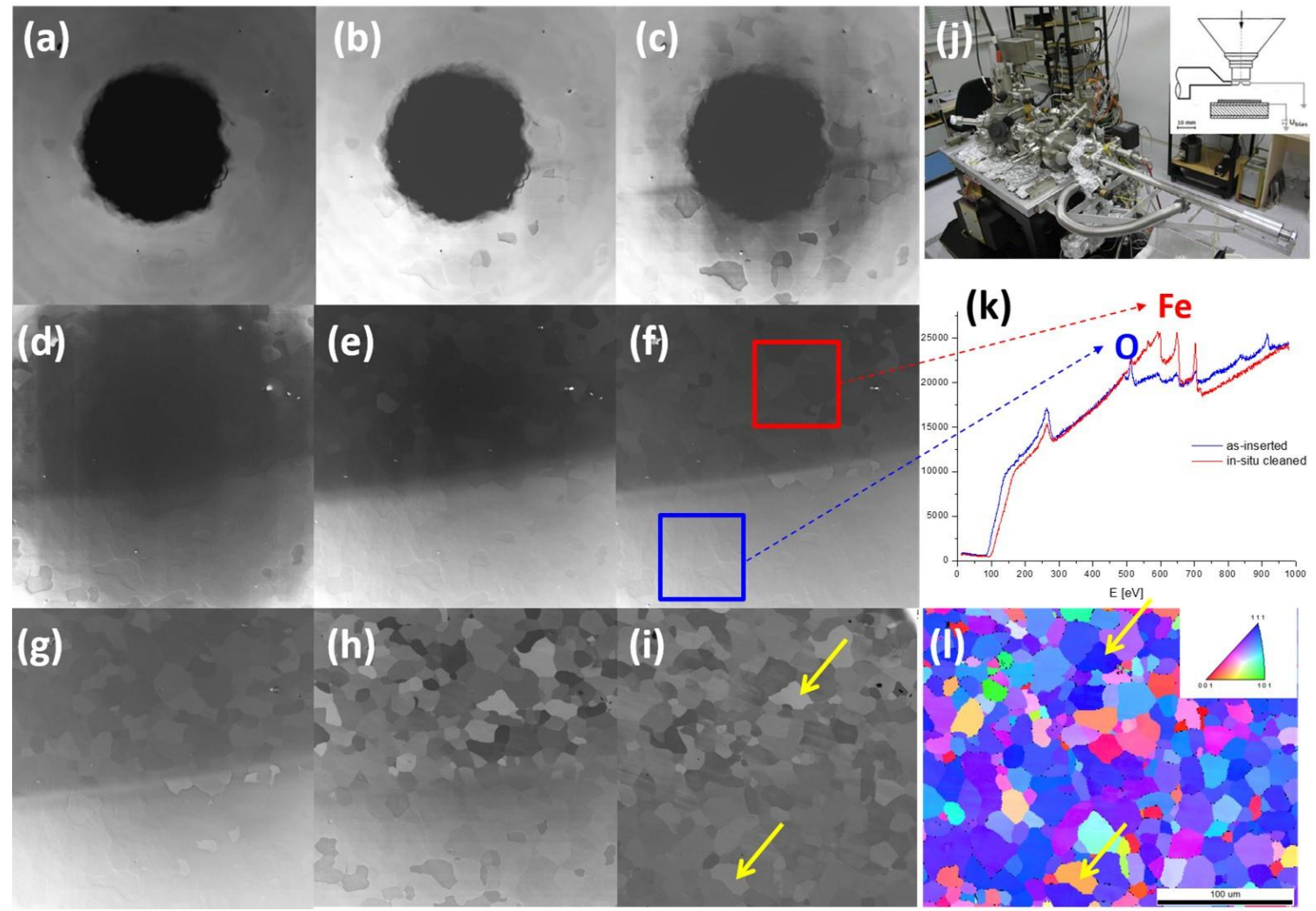

Figure 1. UHV SLEEM micrographs of a mild steel surface (clean/oxidized interface) obtained at various landing energies of the primary electrons (a-i): $0 \mathrm{eV}$ (a), $0.3 \mathrm{eV}$ (b), $0.6 \mathrm{eV}$ (c), 1,2 eV (d), $5.7 \mathrm{eV}$ (e), 12 $\mathrm{eV}$ (f), $50 \mathrm{eV}$ (g), $500 \mathrm{eV}$ (h) and $5 \mathrm{keV}$ (i); together with the UHV SLEEM instrument image + schematic sketch of the cathode lens system (j), Auger spectra collected from the in-situ cleaned (red line) and the as-inserted (blue line) areas (k) and the corresponding EBSD orientation map (l).

(a)

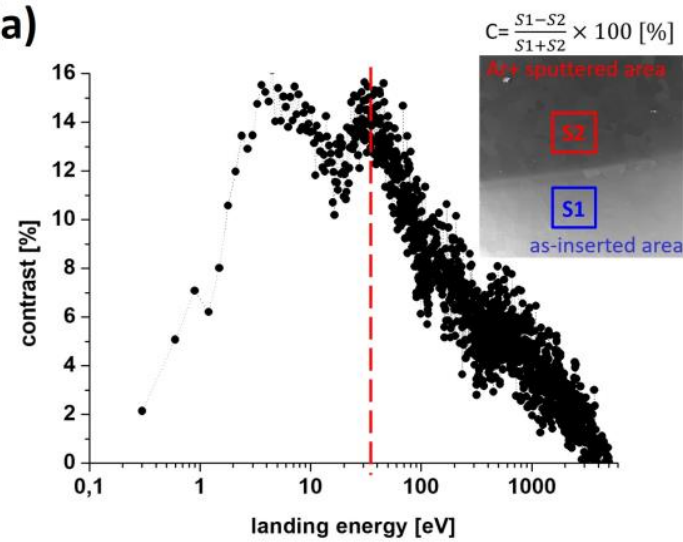

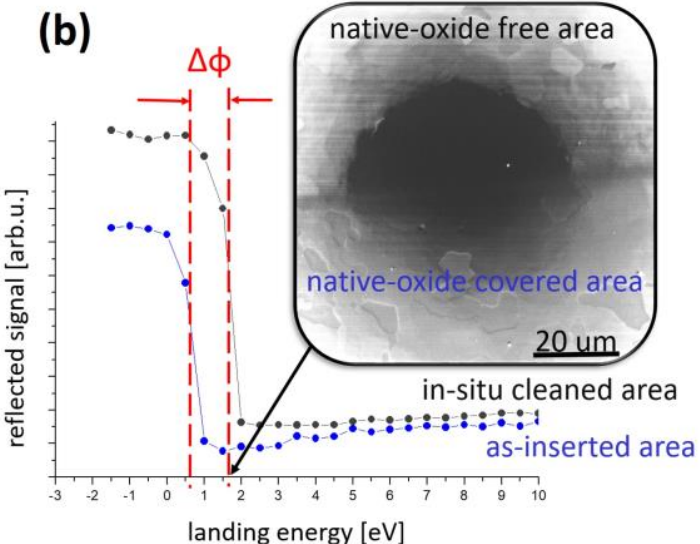

Figure 2. 2 (a): the energy dependence of the contrast between the clean and oxidized areas; 2 (b): the energy dependence of the reflected signal from the native oxide covered (blue points) and the native oxidefree (red points) areas

\section{References}

[1] E. Bauer “Low Energy Electron Microscopy” (Springer, Berlin) 2014. 
[2] I Mullerova et. al. Adv. Imag. Electron Phys. 128 (2003), p. 309-443.

[3] S Mikmekova et. al. Microcopy 64 (2015), p. 347-443.

[4] T Aoyama et. al. Ultramicroscopy 204 (2019), p.1-5.

[5] The authors acknowledge funding from the Technology Agency of the Czech Republic (National Centre of Competence, TN01000008). 